\title{
Computers
}

\& Structures

PERGAMON

\section{An inf-sup test for shell finite elements}

\author{
Klaus-Jürgen Bathe ${ }^{\mathrm{a}, *}$, Alexander Iosilevich ${ }^{\mathrm{a}}$, Dominique Chapelle ${ }^{\mathrm{b}}$ \\ ${ }^{a}$ Massachusetts Institute of Technology, Department of Mechanical Engineering, Cambridge, MA 02139, USA \\ bINRIA-Rocquencourt, BP 105, 78153 Le Chesnay Cedex, France
}

Accepted 10 August 1999

\begin{abstract}
We present an inf-sup test for general mixed shell finite element discretizations. The test is useful in the thorough evaluation of a shell finite element discretization scheme. We apply the test to the MITC shell elements and find that these elements pass the test. (C) 2000 Elsevier Science Ltd. All rights reserved.
\end{abstract}

Keywords: Shell elements; Mixed formulations; MITC elements; Inf-sup condition

\section{Introduction}

It is well-recognized that certain problems in solid and structural mechanics and in fluid mechanics are most effectively solved using mixed finite element formulations. For incompressible analysis of solids (fluids), the displacement (velocity)/pressure-based formulations $(u / p$ elements) are optimal, and for the analysis of plates, the Reissner-Mindlin theory based elements using a mixed interpolation of tensorial components (the MITC plate elements) are very effective [1].

These solid, fluid and plate elements have a strong mathematical foundation [1,2], and experiences with these element discretizations in engineering practice have always been good. The clear advantages of these elements are their reliability (they always give analysis answers that can be relied upon) and computational efficiency.

The key to success of these elements lies in that in each case they satisfy two crucial mathematical con-

\footnotetext{
* Corresponding author. Tel.: +1-617-253-6645; fax: +1617-253-2275.
}

ditions (without the use of any numerical factors like employed in stabilized methods), namely the ellipticity and the relevant inf-sup conditions. The fact that these conditions are satisfied ensures that the finite element discretizations are stable and, moreover, optimal. While it is relatively easy to check whether the ellipticity condition is met, it can be difficult to identify whether the inf-sup condition is satisfied by a specific finite element discretization.

Considering the displacement (velocity)/pressurebased finite element formulations, the analytical proof regarding the satisfaction of the inf-sup condition is available for a number of elements [1,2], and when such proof is not available, a numerical inf-sup test can be easily performed $[1,3]$. While a numerical test cannot be as encompassing as an analytical proof, our experience is that when the numerical test is passed, in fact, the inf-sup condition is satisfied. The numerical inf-sup test is like the patch test (used for non-conforming displacement-based discretizations, see e.g. [1]) usually performed numerically instead of analytically. If the inf-sup condition is satisfied, the element is optimal which means that the convergence rate is the maximum for the interpolations used and is independent of the value of the bulk modulus [1]. 
Considering plate-bending discretizations, a numerical inf-sup test has also been proposed [4]. The test was used to evaluate the MITC plate bending elements $[4,5]$. Since these elements pass the test, we can consider them to provide optimal discretizations, that is, the convergence rate is the maximum possible for the interpolations used and is independent of the plate thickness.

The objective of this paper is to propose an inf-sup test for general shell elements and apply the test to some discretization schemes, in particular, to the MITC shell elements [6,7]. The test is a very useful tool in the search for improved shell elements.

In the following sections of the paper we first review the general mixed finite element formulation that we consider for shell analysis, and the inf-sup condition that distinguishes an optimal discretization scheme. We then derive the inf-sup test and apply the test to displacement-based discretizations and the MITC shell elements. While the displacement-based shell elements, of course, fail the test, the MITC shell elements pass it. The inf-sup test for general shell discretizations can of course also be used to evaluate plate bending elements, and we give some results for this case as well.
Finally, in the last section of the paper we summarize our conclusions regarding this contribution.

\section{Mixed formulation and the inf-sup condition}

We are interested in analyzing the behavior of general three-dimensional shell elements as they are used in engineering practice [1]. These elements are not based on a specific shell theory but are formulated using three-dimensional continuum theory with kinematic and stress assumptions. The "underlying mathematical model" of the finite element discretization was identified in Ref. [8], where it was also shown that the limit problem, as the thickness $t$ of the shell approaches zero, is identical to the limit problem obtained using the Naghdi shell theory. Since our objective is to consider the behavior of the general shell elements as $t \rightarrow 0$, we can base much of our theoretical discussions on using the Naghdi shell theory, while the inf-sup condition employed in our testing is based on using the three-dimensional general shell elements.

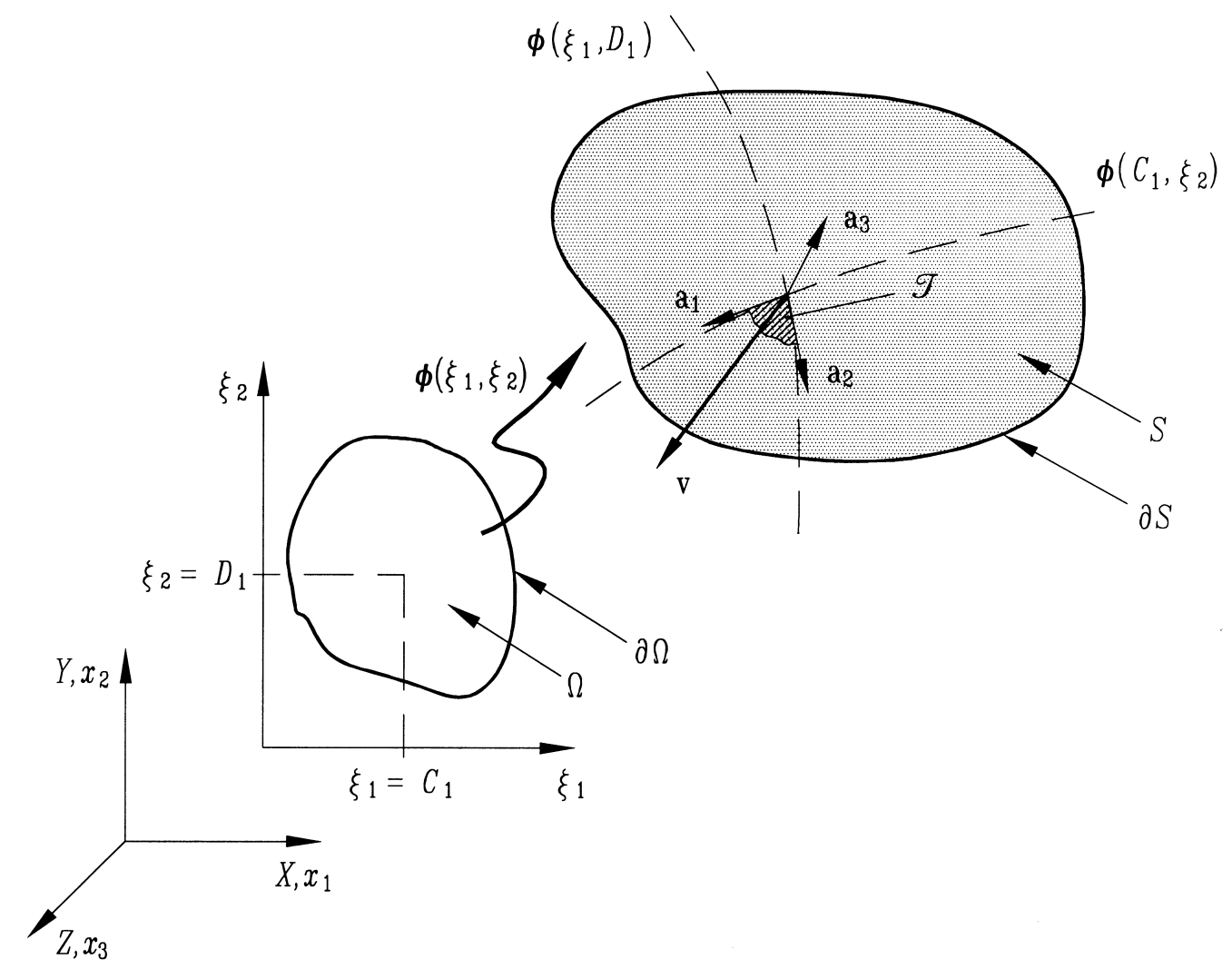

Fig. 1. Definitions pertaining to the shell midsurface. 


\subsection{Naghdi shell model}

We assume that the shell midsurface $S$ is defined by a single chart $\phi$, which is a one-to-one smooth mapping from $\Omega$ into $\mathbb{R}^{3}$, where $\Omega$ denotes an open domain of $\mathbb{R}^{2}$ with a boundary $\partial \Omega$ called "reference domain" and thus $S=\phi(\Omega)$ (see Fig. 1). We now briefly review the classical definitions and notation of differential geometry that we need for our analysis. We use the Einstein convention on the summation of repeated indices, their values ranging in $\{1,2\}$. Let the covariant base of the tangent plane $\mathscr{T}$ be defined by

$\mathbf{a}_{\alpha} \stackrel{\text { def. }}{=} \frac{\partial \phi\left(\xi_{1}, \xi_{2}\right)}{\partial \xi_{\alpha}}$

with the contravariant base given by

$\mathbf{a}_{\alpha} \cdot \mathbf{a}^{\beta}=\delta_{\alpha}^{\beta}$,

where $\delta$ denotes the Kronecker symbol. The unit normal vector is

$\mathbf{a}_{3} \stackrel{\text { def. }}{=} \frac{\mathbf{a}_{1} \times \mathbf{a}_{2}}{\left\|\mathbf{a}_{1} \times \mathbf{a}_{2}\right\|}$.
The first fundamental form of the surface is given by

$a_{\alpha \beta} \stackrel{\text { def. }}{=} \mathbf{a}_{\alpha} \cdot \mathbf{a}_{\beta}$,

or alternatively in contravariant form by

$a^{\alpha \beta} \stackrel{\text { def. }}{=} \mathbf{a}^{\alpha} \cdot \mathbf{a}^{\beta}$.

The second fundamental form is defined by

$b_{\alpha \beta} \stackrel{\text { def. }}{=} \mathbf{a}_{3} \cdot \mathbf{a}_{\alpha, \beta}$.

The following symbol appears in surface measures:

$a \stackrel{\text { def. }}{=}\left\|\mathbf{a}_{1} \times \mathbf{a}_{2}\right\|^{2}=a_{11} a_{22}-a_{12}^{2}$,

and indeed the area of a differential element $\mathrm{d} S$ on the midsurface is calculated as

$\mathrm{d} S=\sqrt{a} \mathrm{~d} \xi_{1} \mathrm{~d} \xi_{2}$.

Referring to Fig. 2, the shell domain can then be described as:

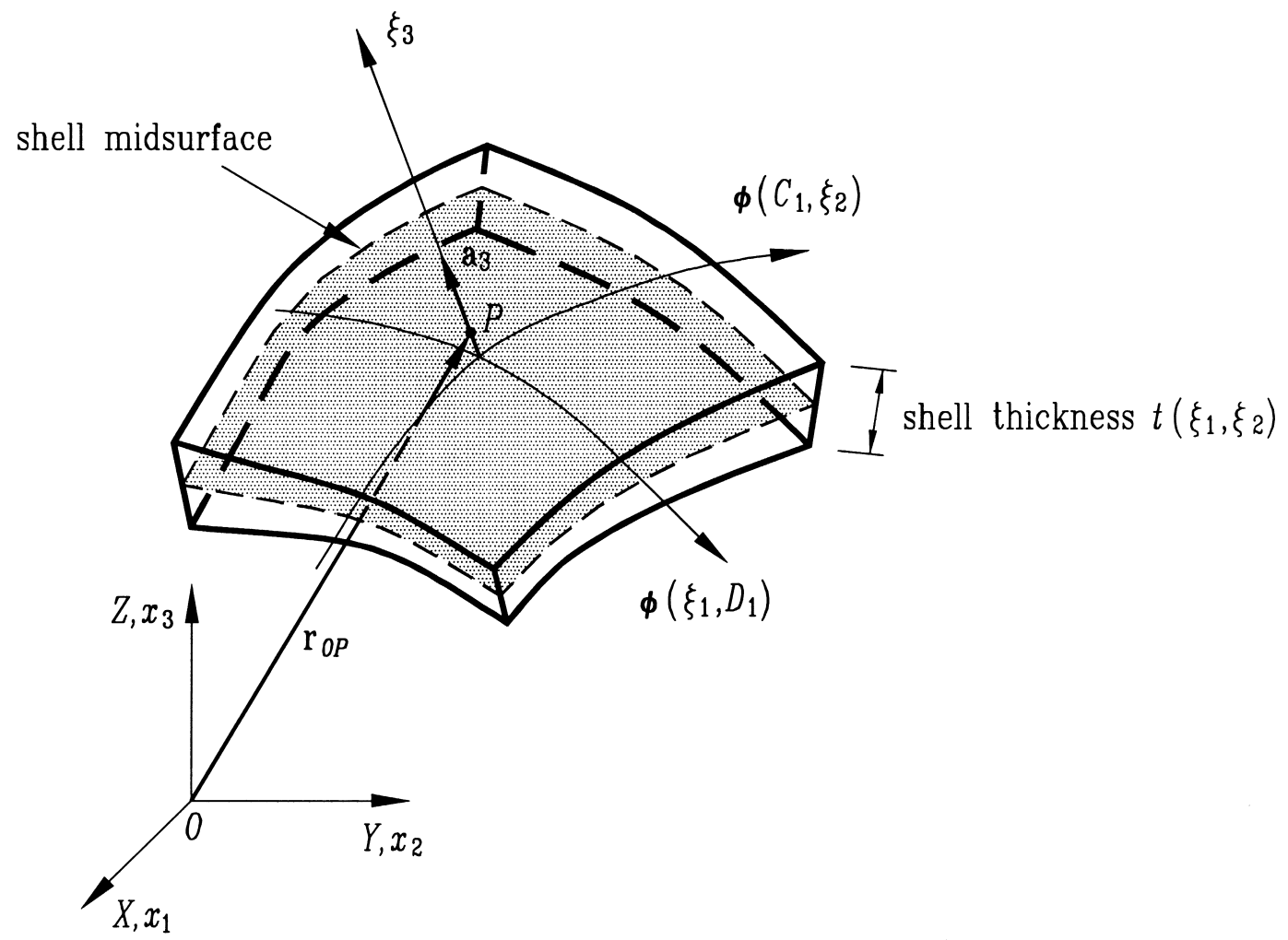

Fig. 2. General shell geometry. 
$\mathscr{S}=\left\{\begin{array}{l}\left\{P \in \mathbb{R}^{3}\right\}, \mathbf{r}_{O P}=\boldsymbol{\phi}\left(\xi_{1}, \xi_{2}\right)+\xi_{3} \mathbf{a}_{3}, \\ \xi_{3} \in\left(-\frac{1}{2} t\left(\xi_{1}, \xi_{2}\right) ; \frac{1}{2} t\left(\xi_{1}, \xi_{2}\right)\right),\end{array}\right.$

where $t$ is the shell thickness.

Let the displacement vector of a material particle on the midsurface of the shell be $\mathbf{v}$, and the rotations of the mid-surface sections be $\eta_{1}$ and $\eta_{2}\left(\eta_{\alpha}\right.$ causing positive displacements into the $\mathbf{a}_{\alpha}$-direction for particles with $\xi_{3}>0$ ).

The variational formulation of the Naghdi shell model is $[9,10]$ :

Find $\mathbf{U}=(\mathbf{u}, \boldsymbol{\beta}) \in \mathscr{U}=\mathscr{V} \times \mathscr{B}$ such that

$t^{3} A(\mathbf{U}, \mathbf{V})+t D(\mathbf{U}, \mathbf{V})=G(\mathbf{V}) \quad \forall \mathbf{V}=(\mathbf{v}, \boldsymbol{\eta}) \in \mathscr{U}$,

where

$\mathscr{V}=\left\{\mathbf{v} \in\left[H^{1}(\Omega)\right]^{3} \cap \mathscr{B} \mathscr{C}_{u}\right\},\|\mathbf{v}\|_{\mathscr{V}}^{2}=\left\|v_{i}\right\|_{H^{1}}^{2} ;$

$\mathscr{B}=\left\{\boldsymbol{\eta} \in\left[H^{1}(\Omega)\right]^{2} \cap \mathscr{B} \mathscr{C}_{\beta}\right\},\|\boldsymbol{\eta}\|_{\mathscr{B}}^{2}=\left\|\eta_{\alpha}\right\|_{H^{1}}^{2} ;$

$\|\mathbf{V}\|_{\mathscr{U}}^{2}=\|\mathbf{v}\|_{\mathscr{V}}^{2}+\|\boldsymbol{\eta}\|_{\mathscr{B}}^{2}$

$\mathscr{B} \mathscr{C}_{u}$ and $\mathscr{B} \mathscr{C}_{\beta}$ are the boundary conditions on displacements and rotations, respectively;

$A(\mathbf{U}, \mathbf{V})=\frac{1}{12} \int_{\Omega} E^{\alpha \beta \lambda \mu} \chi_{\alpha \beta}(\mathbf{U}) \chi_{\lambda \mu}(\mathbf{V}) \mathrm{d} S$

is the bending energy term with bending strains $\chi_{\lambda \mu}$ calculated as

$$
\begin{aligned}
\chi_{\lambda \mu}(\mathbf{V})= & \frac{1}{2}\left[\eta_{\lambda \mid \mu}+\eta_{\mu \mid \lambda}-b_{\lambda}^{\alpha}\left(v_{\alpha \mid \mu}-b_{\alpha \mu} v_{3}\right)-b_{\mu}^{\alpha}\left(v_{\alpha \mid \lambda}\right.\right. \\
& \left.\left.-b_{\alpha \lambda} v_{3}\right)\right]
\end{aligned}
$$

where the vertical bar before a subscript denotes covariant differentiation, and

$$
E^{\alpha \beta \lambda \mu}=\frac{E}{2(1+v)}\left[a^{\alpha \lambda} a^{\beta \mu}+a^{\alpha \mu} a^{\beta \lambda}+\frac{2 v}{(1-v)} a^{\alpha \beta} a^{\lambda \mu}\right]
$$

is the fourth-order tensor of the constitutive law with $E$ and $v$ being the Young's modulus and Poisson's ratio, respectively.

The second bilinear form $D(\mathbf{U}, \mathbf{V})$ can be represented as a sum of the membrane part $D^{m}(\mathbf{u}, \mathbf{v})$ :
$D^{m}(\mathbf{u}, \mathbf{v})=\int_{\Omega} E^{\alpha \beta \lambda \mu} \gamma_{\alpha \beta}(\mathbf{u}) \gamma_{\lambda \mu}(\mathbf{v}) \mathrm{d} S$

where

$\gamma_{\lambda \mu}(\mathbf{v})=\frac{1}{2}\left(v_{\lambda \mid \mu}+v_{\mu \mid \lambda}\right)-b_{\lambda \mu} v_{3}$,

and the shear term $D^{s}(\mathbf{U}, \mathbf{V})$ :

$D^{s}(\mathbf{U}, \mathbf{V})=\int_{\Omega} G^{\alpha \beta} \rho_{\alpha}(\mathbf{U}) \rho_{\beta}(\mathbf{V}) \mathrm{d} S$

with the shear modulus

$G^{\alpha \beta}=\frac{E a^{\alpha \beta}}{2(1+v)}$

and shear strains

$\rho_{\beta}(\mathbf{V})=v_{3, \beta}+b_{\beta}^{\lambda} v_{\lambda}+\eta_{\beta}$.

Finally, $G(\mathbf{V})$ is the potential of the external load $\mathbf{p}$ applied over the midsurface $S$

$G(\mathbf{v})=\int_{\Omega} \mathbf{p} \cdot \mathbf{v} \mathrm{d} S$.

As summarized by Chapelle and Bathe [10], the behavior of a shell structure highly depends on the subspace $\mathscr{U}_{0} \subset \mathscr{U}$, where

$\mathscr{U}_{0}=\{\mathbf{V} \in \mathscr{U} \mid D(\mathbf{V}, \mathbf{V})=0\}$.

This subspace corresponds to pure bending deformations only, that is all shear and membrane strains vanish. We are particularly interested in the case when the thickness $t$ of the shell becomes very small and therefore want to consider the limit problem $t \rightarrow 0$.

If $\mathscr{U}_{0} \neq\{\mathbf{0}\}$, we have the case of a pure-bending-uninhibited shell structure, which corresponds to bendingdominated behavior when $t$ is small.

In order to keep the shell model equations welldefined, we assume for the analysis of the limit problem that

$G(\mathbf{V})=t^{3} F_{b}(\mathbf{V})$,

where $F_{b}(\mathbf{V})$ is a linear form independent of the thickness parameter $t$. Hence, for any finite $t$ Eq. (10) can be restated as

Find $\mathbf{U}_{t} \in \mathscr{U}$, such that

$A\left(\mathbf{U}_{t}, \mathbf{V}\right)+\frac{1}{t^{2}} D\left(\mathbf{U}_{t}, \mathbf{V}\right)=F_{b}(\mathbf{V}) \quad \forall \mathbf{V} \in \mathscr{U}$.

Assuming that the space $\mathscr{U}_{0}$ is non-trivial, the following convergence result can be established (see [10]): 
$\lim _{t \rightarrow 0} \frac{1}{t^{2}} D\left(\mathbf{U}_{t}, \mathbf{U}_{t}\right)=0$

and the solution $\mathbf{U}_{t}$ converges to the solution $\mathbf{U}_{0}^{b} \in \mathscr{U}_{0}$ of the following limit problem:

$A\left(\mathbf{U}_{0}^{b}, \mathbf{V}\right)=F_{b}(\mathbf{V}) \quad \forall \mathbf{V} \in \mathscr{U}_{0}$

If, however, $\mathscr{U}_{0}=\{\mathbf{0}\}$, we have the case of a pure-bending-inhibited shell structure, which corresponds to membrane-dominated behavior when $t$ is small. To consider the limit problem, the proper scaling for the load term is then

$G(\mathbf{V})=t F_{m}(\mathbf{V})$,

and we can rewrite Eq. (10) as

Find $\mathbf{U}_{t} \in \mathscr{U}$, such that

$t^{2} A\left(\mathbf{U}_{t}, \mathbf{V}\right)+D\left(\mathbf{U}_{t}, \mathbf{V}\right)=F_{m}(\mathbf{V}) \quad \forall \mathbf{V} \in \mathscr{U}$.

As in the previous case, we can establish (see [10] and references therein) that

$\lim _{t \rightarrow 0} t^{2} A\left(\mathbf{U}_{t}, \mathbf{U}_{t}\right)=0$

and $\mathbf{U}_{t}$ converges to the solution $\mathbf{U}_{0}^{m} \in \mathscr{W}$ of the following limit (membrane) problem

$D\left(\mathbf{U}_{0}^{m}, \mathbf{V}\right)=F_{m}(\mathbf{V}) \quad \forall \mathbf{V} \in \mathscr{W}$,

where $\mathscr{W}$ is the space of all displacements $\mathbf{V}$ for which $D(\mathbf{V}, \mathbf{V})$ is finite.

This convergence property holds true provided, of course, that the loading term satisfies a regularity requirement

$\left|F_{m}(\mathbf{V})\right|^{2} \leq c D(\mathbf{V}, \mathbf{V}) \quad \forall \mathbf{V} \in \mathscr{W}$

with $c$ being a finite positive constant [10].

The finite element solution of the shell problem can now be sought by choosing a discrete space $\mathscr{U}^{h} \subset \mathscr{U}$, and considering a conforming approximation of the variational problem in Eq. (10):

Find $\mathbf{U}^{h} \in \mathscr{U}^{h}$, such that

$t^{3} A\left(\mathbf{U}^{h}, \mathbf{V}\right)+t D\left(\mathbf{U}^{h}, \mathbf{V}\right)=G(\mathbf{V}) \quad \forall \mathbf{V} \in \mathscr{U}^{h}$

To analyze the performance of the finite element discretization, we consider the two limiting states corresponding to the membrane-dominated (inhibited) and bending-dominated (non-inhibited) cases. In the inhibited case, the pure displacement-based conforming finite element discretization works well in that the discretization remains uniformly convergent, independent of the shell thickness $t$, see [10]. However, considering the non-inhibited case, the behavior referred to as "locking" occurs as $t \rightarrow 0$. Namely, in this case the variational problem considered is:

Find $\mathbf{U}_{t}^{h} \in \mathscr{U}^{h}$, such that

$A\left(\mathbf{U}_{t}^{h}, \mathbf{V}\right)+\frac{1}{t^{2}} D\left(\mathbf{U}_{t}^{h}, \mathbf{V}\right)=F_{b}(\mathbf{V}) \quad \forall \mathbf{V} \in \mathscr{U}^{h}$,

and the convergence is highly influenced by how rich $\mathscr{U}^{h}$ is in $\mathscr{U}_{0}$. In the worst case $\mathscr{U}^{h} \cap \mathscr{U}_{0}=\{\boldsymbol{0}\}$ leading to total loss of convergence

$\lim _{t \rightarrow 0} \mathbf{U}_{t}^{h}=\mathbf{0} \neq \lim _{t \rightarrow 0} \mathbf{U}_{t}=\mathbf{U}_{0}^{b}$

In practice, extremely fine meshes are needed for an accurate solution when $t$ is small, and the displacement-based finite element procedure becomes unpractical. The remedy is to use an appropriate mixed formulation. The aim with this approach is to interpolate displacements and strains (or stresses) in such a manner as to have no locking of the discretization in the bending-dominated case for any value of thickness $t$, and to have, as well, a uniformly good behavior for the membrane-dominated case. Ideally, the rate of convergence would be optimal, independent of whether a bending-dominated or membrane-dominated problem is considered and independent of the shell thickness.

The key to reaching this optimal behavior is to use in the mixed formulation "well-balanced" interpolations and to satisfy the inf-sup condition for the bending-dominated situation.

\subsection{Mixed formulation}

The general mixed formulation that we wish to consider is given by:

$$
\begin{aligned}
& \text { Find } \mathbf{U}^{h}=\left(\mathbf{u}^{h}, \boldsymbol{\beta}^{h}\right) \in \mathscr{U}^{h}=\left(\mathscr{V}^{h}, \mathscr{B}^{h}\right) \text { and } \\
& \mathbf{E}^{h}=\left\{\varepsilon_{i j}^{h}\right\} \in \mathscr{E}^{h}, \text { such that } \\
& \left\{\begin{array}{l}
t^{3} \tilde{A}\left(\mathbf{E}^{h}, \boldsymbol{\varepsilon}(\mathbf{V})\right)+t \tilde{D}\left(\mathbf{E}^{h}, \boldsymbol{\varepsilon}(\mathbf{V})\right)=G(\mathbf{V}) \\
\tilde{A}\left(\mathbf{E}^{h}-\boldsymbol{\varepsilon}\left(\mathbf{U}^{h}\right), \boldsymbol{\Psi}\right)=0 \\
\tilde{D}\left(\mathbf{E}^{h}-\boldsymbol{\varepsilon}\left(\mathbf{U}^{h}\right), \boldsymbol{\Psi}\right)=0 \quad \forall \mathbf{V} \in \mathscr{U}^{h}, \boldsymbol{\Psi}=\left\{\psi_{i j}\right\} \in \mathscr{E}^{h},
\end{array}\right.
\end{aligned}
$$

where

$\mathscr{E}^{h}=\left\{\boldsymbol{\Psi}=\left\{\psi_{i j}\right\}, \psi_{i j} \in \mathscr{E}_{i j}^{h}\right\}$,

and $\varepsilon(\cdot)$ is the linear strain operator. Here $\mathscr{E}^{h}$ is the subspace of assumed strains and includes assumed bending, membrane and shear strains, and the bilinear 
forms used are defined so that

$$
\begin{aligned}
& \tilde{A}(\boldsymbol{\varepsilon}(\mathbf{U}), \boldsymbol{\varepsilon}(\mathbf{V}))=A(\mathbf{U}, \mathbf{V}) \\
& \tilde{D}(\boldsymbol{\varepsilon}(\mathbf{U}), \boldsymbol{\varepsilon}(\mathbf{V}))=D(\mathbf{U}, \mathbf{V})
\end{aligned}
$$

Included in the formulation of Eq. (34) are, in particular, the MITC shell elements [6,7]. The displacement and strain interpolations of the elements are summarized in Appendix A. The formulation can be derived from the Hellinger-Reissner variational principle and therefore also from the $\mathrm{Hu}-\mathrm{Washizu}$ variational principle [1]. We note that using the MITC formulation, the element matrices are evaluated by tying the strain interpolations to the displacement interpolations at the tying points given in Appendix A. This tying procedure ensures the integration process implied in Eq. (34), see [11].

The performance of the MITC shell elements was studied in [11], where we have identified that the elements perform well in membrane- and bending-dominated problems. The elements satisfy the ellipticity condition (which can be easily checked, see below), and the results given in Ref. [11] suggest that the infsup condition holds true as well. However, an explicit study of this condition was not given, and this is accomplished below.

\subsection{Stability and optimality of mixed formulation, abstract results}

We consider the bending-dominated case and rewrite the MITC shell formulation in the general classical form:

Find $\mathbf{U}^{h} \in \mathscr{U}^{h}$ and $\tilde{\mathbf{E}}^{h} \in \tilde{\mathscr{E}}^{h}$ such that

$$
\left\{\begin{array}{l}
\tilde{A}\left(\boldsymbol{\varepsilon}^{\mathrm{AS}}\left(\mathbf{U}^{h}\right), \boldsymbol{\varepsilon}^{\mathrm{AS}}(\mathbf{V})\right)+B\left(\tilde{\mathbf{E}}^{h}, \mathbf{V}\right)=F_{b}(\mathbf{V}) \quad \forall \mathbf{V} \in \mathscr{U}^{h} \\
B\left(\boldsymbol{\Psi}, \mathbf{U}^{h}\right)-t^{2} C\left(\tilde{\mathbf{E}}^{h}, \boldsymbol{\Psi}\right)=0 \quad \forall \boldsymbol{\Psi} \in \tilde{\mathscr{E}}^{h},
\end{array}\right.
$$

where $\boldsymbol{\varepsilon}^{\mathrm{AS}}$ is defined by the tying of the bending strains in Eq. (34), $\tilde{\mathbf{E}}^{h}$ is obtained from $\mathbf{E}^{h}$ by retaining only the membrane and shear components of the strains divided by $t^{2}, \mathscr{E}^{-h}$ corresponds to that space, and

$$
\begin{aligned}
& B\left(\tilde{\mathbf{E}}^{h}, \mathbf{V}\right)=\tilde{D}\left(\tilde{\mathbf{E}}^{h}, \boldsymbol{\varepsilon}(\mathbf{V})\right) \\
& C\left(\tilde{\mathbf{E}}^{h}, \boldsymbol{\Psi}\right)=\tilde{D}\left(\tilde{\mathbf{E}}^{h}, \boldsymbol{\Psi}\right) .
\end{aligned}
$$

A mixed formulation of this type should satisfy the fol- lowing two classical conditions in order to have an optimal procedure $[1,2,12]$ :

(i) Ellipticity of $\tilde{A}(\cdot, \cdot)$ on the null-space of $B(\cdot, \cdot)$ :

$\exists$ a constant $\alpha>0$ such that:

$\tilde{A}\left(\boldsymbol{\varepsilon}^{\mathrm{AS}}(\mathbf{V}), \boldsymbol{\varepsilon}^{\mathrm{AS}}(\mathbf{V})\right) \geq \alpha\|\mathbf{V}\|_{\mathscr{U}} \quad \forall \mathbf{V} \in \mathscr{U}_{0}^{h}$,

where

$\mathscr{U}_{0}^{h}=\left\{\mathbf{V} \in \mathscr{U} \mid B(\boldsymbol{\Psi}, \mathbf{V})=0 \forall \boldsymbol{\Psi} \in \tilde{\mathscr{E}}^{h}\right\}$

is the null space of $B(\cdot, \cdot)$.

This condition is a basic solvability condition of the formulation. It is relatively easy to check whether a given formulation satisfies this condition: every element in the discretization should contain the six physical rigid body modes and no spurious zero energy modes. Finite element discretizations that violate this condition should not be used in engineering practice because the formulations are unreliable and may yield misleading results [1].

\section{(ii) The inf-sup condition:}

$\exists$ a constant $\beta>0$ independent of the shell thickness $t$, such that

$$
\sup _{\mathbf{V} \in \mathscr{U}^{h}} \frac{B(\boldsymbol{\Psi}, \mathbf{V})}{\|\mathbf{V}\|_{\mathscr{U}}} \geq \beta \sup _{\mathbf{V} \in \mathscr{U}} \frac{B(\boldsymbol{\Psi}, \mathbf{V})}{\|\mathbf{V}\|_{\mathscr{U}}} \quad \forall \boldsymbol{\Psi} \in \tilde{\mathscr{E}}^{h} .
$$

This inf-sup condition follows from the Banach theorems $[2,13]$. Note that since the supremum on the right-hand side of Eq. (41) is an abstract semi-norm on the strain field $\boldsymbol{\Psi}$, Eq. (41) corresponds to the very general form of the inf-sup condition. In some specific formulations this semi-norm can be written in a more explicit form, such as in analysis of incompressible media or plate bending, see [2-4].

In addition, in order to obtain an optimal error bound, the consistency error due to the use of $\tilde{A}\left(\boldsymbol{\varepsilon}^{\mathrm{AS}}\left(\mathbf{U}^{h}\right), \boldsymbol{\varepsilon}^{\mathrm{AS}}(\mathbf{V})\right)$ instead of $A\left(\mathbf{U}^{h}, \mathbf{V}\right)$ should be properly controlled. To mathematically prove that consistency holds is, however, a more standard procedure and we do not discuss this issue further in this paper.

When the two conditions given by Eqs. (39) and (41) hold (and provided the consistency error is properly controlled), the following optimal error bound can be established [2]:

$$
\begin{aligned}
& \left\|\mathbf{U}-\mathbf{U}^{h}\right\|_{\mathscr{U}}+\left\|\tilde{\mathbf{E}}-\tilde{\mathbf{E}}^{h}\right\|_{\mathscr{E}} \leq c \inf _{\mathbf{V} \in \mathscr{U}^{h}, \boldsymbol{\Psi} \in \tilde{E}^{-h}}\left(\|\mathbf{U}-\mathbf{V}\|_{\mathscr{U}}\right. \\
& \left.+\|\tilde{\mathbf{E}}-\boldsymbol{\Psi}\|_{\mathscr{E}}\right)
\end{aligned}
$$

with the constant $c$ independent of the shell thickness 
$t$, and $\|\cdot\|_{\mathscr{E}}$ denoting the semi-norm on the right-hand side of Eq. (41). The convergence properties of the finite element spaces ensure that the optimal rate of convergence is obtained for sufficiently smooth solutions, i.e.,

$\left\|\mathbf{U}-\mathbf{U}^{h}\right\|_{\mathscr{U}}+\left\|\tilde{\mathbf{E}}-\tilde{\mathbf{E}}^{h}\right\|_{\mathscr{E}} \sim c h^{k}$

where $k$ is the polynomial order of the chosen finite element interpolation.

While for a given formulation, it is easy to check (analytically or numerically) whether the ellipticity condition is satisfied, it is difficult to identify whether the inf-sup condition is satisfied, because the geometry and boundary conditions are crucial ingredients and any possible problems with any sequence of meshes should be considered. This can only be achieved analytically. Arnold and Brezzi constructed an element family for which analytically the ellipticity and inf-sup conditions are satisfied (under very restrictive geometry assumptions) [13]. However, the proposed elements require that a numerical constant be chosen and do not perform well in membrane-dominated problems [14]. Another approach to use is to formulate mixed element discretizations that by-pass the inf-sup condition [15]. However, these formulations also require numerical constants, are not applicable to membrane-dominated situations and have therefore only limited practical value.

When an analytical treatment of the inf-sup condition is out of reach, a numerical test is of value, as discussed in $[1,3]$.

\subsection{Mixed formulation for continuum-based shell elements}

Having considered the classical mixed formulation based on the Naghdi shell theory, we now address the general continuum-based shell elements [1].

Let us denote the internal work corresponding to the three-dimensional (3D) strain fields $\mathbf{E}$ and $\boldsymbol{\Psi}$ calculated using the linear elasticity principles as $A^{3 \mathrm{D}}(\mathbf{E}, \boldsymbol{\Psi})$. The continuum-based shell finite element model can then be written as

$$
\begin{aligned}
& \text { Find } \mathbf{U}^{h} \in \mathscr{U}^{h} \text { and } \mathbf{E}^{h} \in \mathscr{E}^{h} \\
& \text { such that }\left\{\begin{array}{l}
A^{3 \mathrm{D}}\left(\mathbf{E}^{h}, \boldsymbol{\varepsilon}(\mathbf{V})\right)=G(\mathbf{V}) \quad \forall \mathbf{V} \in \mathscr{U}^{h} \\
A^{3 \mathrm{D}}\left(\mathbf{E}^{h}-\boldsymbol{\varepsilon}\left(\mathbf{U}^{h}\right), \boldsymbol{\Psi}\right)=0 \quad \forall \boldsymbol{\Psi} \in \mathscr{E}^{h} .
\end{array}\right.
\end{aligned}
$$

The formulation defined in Eq. (44) involves a twodimensional variational shell model that is not explicitly formulated (see [8]). Therefore, a complete mathematical analysis of this formulation, in particular the asymptotic behavior of the solution and possible locking difficulties, is very difficult to achieve.

However, considering bending-dominated shell pro- blems we know that the critical part of the formulation is the term that corresponds to the membrane and shear energy, see Section 2.3. It can be seen that this term can be simply calculated by considering the 3D energy density and integrating it over the midsurface of the shell times the thickness (and not through the thickness). We call $B(\boldsymbol{\Psi}, \mathbf{V})$ the internal work thus obtained for a displacement field $\mathbf{V}$ and a strain field $\boldsymbol{\Psi}$. Also, we obtain $C(\boldsymbol{\Psi}, \boldsymbol{\Theta})$ as the membrane and shear work corresponding to two strain fields $\boldsymbol{\Psi}$ and $\boldsymbol{\Theta}$. Hence,

$B(\boldsymbol{\Psi}, \mathbf{V})=C(\boldsymbol{\Psi}, \boldsymbol{\varepsilon}(\mathbf{V}))$,

and now the inf-sup condition of Eq. (41) can be directly applied.

\section{The inf-sup test}

In order to construct a numerical inf-sup test, one possibility is to substitute the semi-norm on the righthand side of Eq. (41) with

$\|\boldsymbol{\Psi}\|_{\mathscr{E}_{h / 2 n}}=\sup _{\mathbf{V} \in \mathscr{U}_{h / 2 n}} \frac{B(\boldsymbol{\Psi}, \mathbf{V})}{\|\mathbf{V}\|_{\mathscr{U}}}$,

where $\mathscr{U}_{h / 2 n}$ is the finite element space obtained by subdividing the mesh corresponding to $\mathscr{U}^{h}$ into $2 n \times 2 n$ sub-elements for each element with $n$ sufficiently large. This is, in fact, the approach for plate bending formulations followed in Ref. [4]. However, the computer implementation of this procedure is complex and expensive. To circumvent this difficulty, we follow a different strategy, using simply the $L^{2}$-norm instead of the semi-norm appearing in Eq. (41) or Eq. (46).

\subsection{The inf-sup condition in the $L^{2}$-norm}

Define

$$
\begin{gathered}
\operatorname{Im} B^{h}=\left\{\boldsymbol{\Theta} \in \mathscr{E}^{h}, \text { such that } \exists \mathbf{W} \in \mathscr{U}^{h} \mid B(\boldsymbol{\Psi}, \mathbf{W})\right. \\
\left.=C(\boldsymbol{\Psi}, \boldsymbol{\Theta}), \quad \forall \boldsymbol{\Psi} \in \mathscr{E}^{h}\right\} .
\end{gathered}
$$

The test that we propose is based on the following result:

Proposition 1. Assume that

$$
\sup _{\mathbf{V} \in \mathscr{U}^{h}} \frac{B(\boldsymbol{\Psi}, \mathbf{V})}{\|\mathbf{V}\|_{\mathscr{U}}} \geq \beta^{\prime}\|\boldsymbol{\Psi}\|_{L^{2}}, \quad \forall \boldsymbol{\Psi} \in \operatorname{Im} B^{h},
$$

then 
$\sup _{\mathbf{V} \in \mathscr{U}^{h}} \frac{B(\boldsymbol{\Psi}, \mathbf{V})}{\|\mathbf{V}\|_{\mathscr{U}}} \geq \tilde{\beta} \sup _{\mathbf{V} \in \mathscr{U}} \frac{B(\boldsymbol{\Psi}, \mathbf{V})}{\|\mathbf{V}\|_{\mathscr{U}}}, \quad \forall \boldsymbol{\Psi} \in \operatorname{Im} B^{h}$

where $\beta^{\prime}$ and $\tilde{\beta}$ are strictly positive constants.

The proof of this proposition is immediate, recalling that $B(\boldsymbol{\Psi}, \mathbf{V})$ must be bounded, namely

$B(\boldsymbol{\Psi}, \mathbf{V}) \leq c\|\boldsymbol{\Psi}\|_{L^{2}}\|\mathbf{V}\|_{\mathscr{U}}, \quad \forall(\boldsymbol{\Psi}, \mathbf{V}) \in L^{2} \times \mathscr{U}$,

hence

$$
\sup _{\mathbf{V} \in \mathscr{U}} \frac{B(\boldsymbol{\Psi}, \mathbf{V})}{\|\mathbf{V}\|_{\mathscr{U}}} \leq c\|\boldsymbol{\Psi}\|_{L^{2}}
$$

Note that it is $\operatorname{Im} B^{h}$ that appears in Eq. (49) instead of $\mathscr{E}^{h}$ in the inf-sup condition (41). Hence, in general, the two conditions are not equivalent, although they are closely related as shown in the following proposition.

Proposition 2. Consider the assumption

(A) For any $\boldsymbol{\Psi}$ in $\mathscr{E}^{\text {h }}$ such that

$B(\boldsymbol{\Psi}, \mathbf{V})=0, \quad \forall \mathbf{V} \in \mathscr{U}^{h}$,

it also holds that

$B(\boldsymbol{\Psi}, \mathbf{V})=0, \quad \forall \mathbf{V} \in \mathscr{U}$

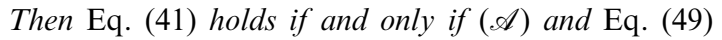
both hold.

The proof of this proposition is given in Appendix B. Saying that $(\mathscr{A})$ does not hold means that there exists at least one assumed strain field $\boldsymbol{\Psi}$ which satisfies Eq. (52), but not Eq. (53). Such a field may be called a "spurious shear/membrane mode", as it is indeed similar to a "spurious pressure mode" in incompressible analysis, see $[1,3]$. Therefore, we can see that Eq. (41) is a stronger condition than Eq. (49) in that it also ensures that there are no spurious shear/membrane modes. However, as noted in [1,3], the existence of such spurious modes does not affect the results unless these modes are activated by prescribed displacement boundary conditions. Hence, condition (49) is sufficient for most purposes ${ }^{1}$.

Of course, ideally, a mixed formulation does not exhibit spurious shear/membrane modes, and for the

\footnotetext{
${ }^{1}$ It should be pointed out that the discussed "spurious pressure modes" and "spurious shear/membrane modes" are not like the spurious modes due to reduced integration, which can be detrimental [1]. Hence, we do not use reduced integration.
}

elements and test problems we have considered in our study spurious modes were not encountered (and hence in each case $\operatorname{Im} B^{h} \equiv \mathscr{E}^{h}$, see Section 4). However, in general, mixed element discretizations may include spurious shear/membrane modes and for this reason we include their occurrence in our theoretical developments.

The objective of the test that we now propose is to identify whether or not Eq. (48) holds. Indeed, if Eq. (48) holds, then Eq. (49) holds too, hence convergence is guaranteed independently of the thickness parameter, provided the other condition (namely ellipticity) is also satisfied. Note that, if a finite element scheme fails the test, no conclusion can be drawn since Eqs. (48) and (49) are not equivalent. Yet, we consider this procedure as valuable since it indicates, at a reasonable computational cost, that some finite elements (which pass the test) are good for bendingdominated thin shell problems.

\subsection{Calculation of the inf-sup value}

Our test relies on the numerical evaluation of the coefficient $\beta^{\prime}$ appearing in Eq. (48), i.e. we need to be able to calculate the quantity

$$
\inf _{\boldsymbol{\Psi} \in \operatorname{Im} B^{h}} \sup _{\mathbf{V} \in \mathscr{U}^{h}} \frac{B(\boldsymbol{\Psi}, \mathbf{V})}{\|\mathbf{V}\|_{\mathscr{U}}\|\boldsymbol{\Psi}\|_{L^{2}}} .
$$

In fact, in the denominator of this quantity we will use $\sqrt{C(\boldsymbol{\Psi}, \boldsymbol{\Psi})}$ instead of the usual $L^{2}$-norm, which is, of course, equivalent.

For a displacement $\mathbf{V}$, we define $\boldsymbol{\varepsilon}^{\mathrm{AS}}(\mathbf{V})$, the assumed strain associated with $\mathbf{V}$, as the unique assumed strain field such that

$C(\boldsymbol{\varepsilon}(\mathbf{V}), \boldsymbol{\Psi})=C\left(\varepsilon^{\mathrm{AS}}(\mathbf{V}), \boldsymbol{\Psi}\right), \quad \forall \boldsymbol{\Psi} \in \mathscr{E}^{h}$.

This means that $\boldsymbol{\varepsilon}^{\mathrm{AS}}(\mathbf{V})$ is the $L^{2}$-projection of $\boldsymbol{\varepsilon}(\mathbf{V})$ onto the space $\mathscr{E}^{h}$.

We now introduce the modified stiffness matrix corresponding to the shear and membrane deformation energy, $\tilde{\mathbf{K}}$, defined by

$\hat{\mathbf{U}}^{\mathrm{T}} \tilde{\mathbf{K}} \hat{\mathbf{V}}=C\left(\boldsymbol{\varepsilon}^{\mathrm{AS}}(\mathbf{U}), \boldsymbol{\varepsilon}^{\mathrm{AS}}(\mathbf{V})\right)$,

where $\hat{\mathbf{U}}$ and $\hat{\mathbf{V}}$ are the nodal displacement vectors corresponding to displacement fields $\mathbf{U}$ and $\mathbf{V}$, respectively. We also define the norm matrix, $\mathbf{S}$, by

$$
\hat{\mathbf{V}}^{\mathrm{T}} \mathbf{S} \hat{\mathbf{V}}=\|\mathbf{V}\|_{\mathscr{U}}^{2}
$$

The evaluation of the inf-sup quantity is based on the following result, proved in Refs. [1,3].

Proposition 3. Define $\lambda_{\min }$ as the smallest non-zero 
eigenvalue of the problem

$\tilde{\mathbf{K}} \hat{\mathbf{V}}=\lambda \mathbf{S} \hat{\mathbf{V}}$.

Then we have

$\inf _{\boldsymbol{\Psi} \in \operatorname{Im} B^{h}} \sup _{\mathbf{V} \in \mathscr{U}^{h}} \frac{B(\boldsymbol{\Psi}, \mathbf{V})}{\|\mathbf{V}\|_{\mathscr{U}} \sqrt{C(\boldsymbol{\Psi}, \boldsymbol{\Psi})}}=\sqrt{\lambda_{\min }}$.

\subsection{Effective implementation of the inf-sup test}

Based on the above considerations, we propose a simple and effective computer implementation of the inf-sup test for general shell elements.

1. In order to perform the inf-sup analysis of a particular finite element, a suitable set of well-chosen bending-dominated problems should be selected. The convergence studies given in Ref. [11] indicate that the free cylinder and the hyperbolic paraboloid shell problems can be used as good tests. In addition, a sequence of $K$ finite element meshes should be chosen, with decreasing characteristic element size $h^{k}(k=1, \ldots, K)$, and preferably with the element sides not aligned on the asymptotic lines of the mid-surface (see Ref. [10]).

2. For every mesh $k$ in the chosen sequence, establish the stiffness matrix $\tilde{\mathbf{K}}^{k}$ and norm matrix $\mathbf{S}^{k}$ and cal- culate the smallest non-zero eigenvalue $\lambda_{\min }^{k}$ of the generalized eigenvalue problem

$$
\tilde{\mathbf{K}}^{k} \hat{\mathbf{V}}=\lambda^{k} \mathbf{S}^{k} \hat{\mathbf{V}}
$$

3. Plot $\log \left(\lambda_{\min }^{k}\right)$ versus $\log \left(h^{k}\right)$. If the curve clearly flattens out as $h^{k}$ decreases, meaning that the $\lambda_{\text {min }}$ values stabilize at some positive level, then we consider that Condition (48) is satisfied.

4. If the right behavior is observed for all test problems, we conclude that the element passes the infsup test.

\section{Numerical results}

In this section we report the results of the numerical inf-sup test considering a number of bending-dominated problems using displacement-based and mixed interpolated general shell elements. The displacementbased elements are the nine-node (QUAD9) and sixteen-node (QUAD16) quadrilateral and six-node triangular (TRI6) elements. All element matrices are evaluated using full numerical integration [1]. The material constants used are: Young's modulus $E=1.0$ and Poisson ratio $v=0.3$. As expected, the eigenvalue problem of Eq. (60) has many zero eigenvalues, the

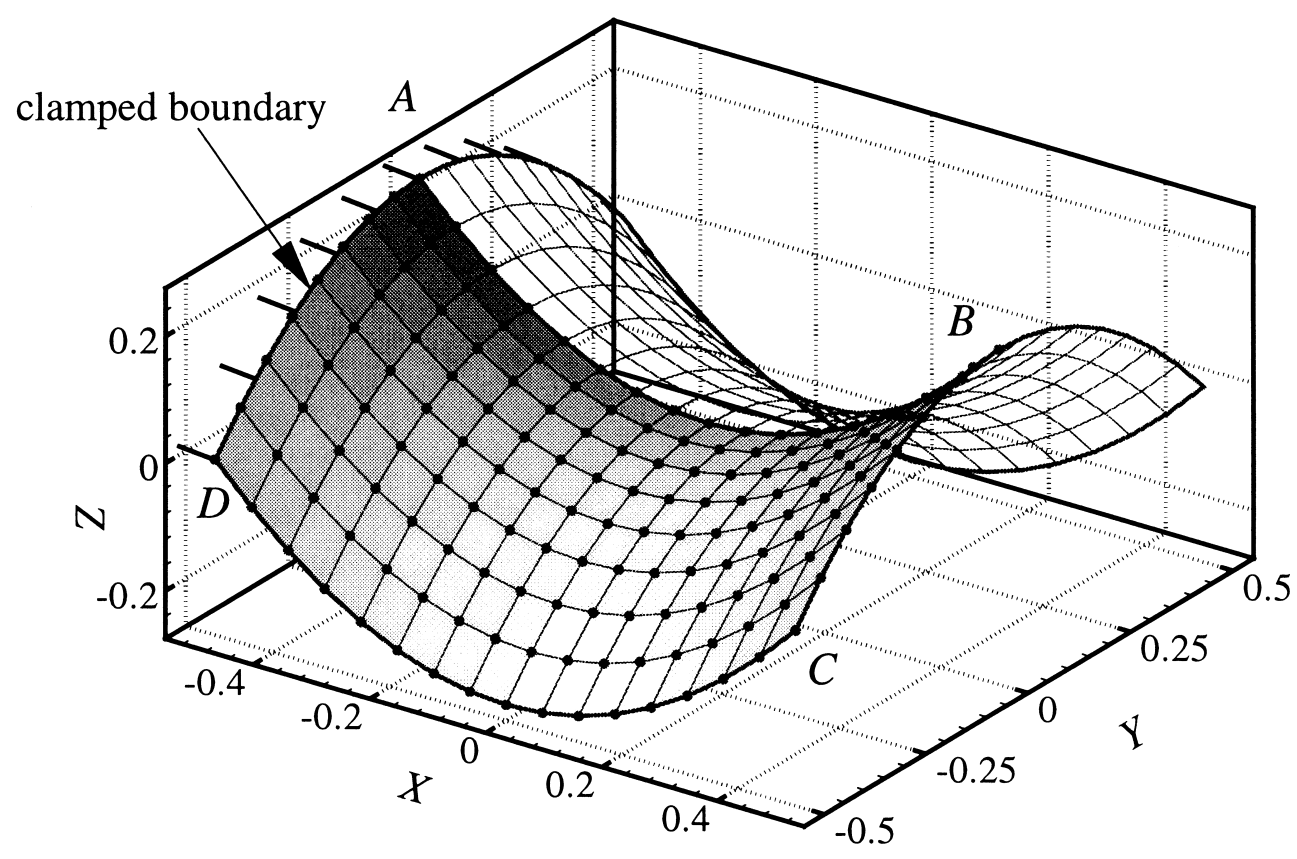

Fig. 3. Partly clamped hyperbolic paraboloid. A $16 \times 8$ mesh of four-node elements is shown. 
number of which can be calculated [1]. We have found that in all tests reported below, no spurious zero eigenvalues were encountered. Note that while the values of the first nonzero eigenvalue reported in the figures below are very small due to the selected Young's modulus, the computed eigenvalues declared as zero were smaller by about $4-5$ orders of magnitude.

\subsection{Analysis of hyperbolic paraboloid}

We apply the inf-sup test methodology developed above and consider the partly clamped hyperbolic paraboloid problem shown in Fig. 3. We choose sequences of uniform meshes of quadrilateral and triangular elements.

Fig. 4 reports the convergence of the smallest eigenvalue $\lambda_{\min }$ versus the mesh density indicator $1 / N$, where $N$ is the number of subdivisions along the $X$-axis.

We clearly see that the displacement-based elements fail the inf-sup test - their smallest eigenvalues steadily converge to zero as the meshes are refined. On the other hand, the mixed-interpolated MITC elements pass the test - their smallest nonzero eigenvalues stabilize at the level of $\sim 10^{-7}$.

\subsection{Analysis of free cylindrical shell}

We consider the free cylindrical shell shown in Fig. 5 using uniform meshes of triangular and quadrilateral elements.

Fig. 6 displays the convergence of the smallest nonzero eigenvalue versus the mesh density indicator $1 / N$. Like for the hyperbolic paraboloid problem, the $\lambda_{\text {min }}$ values of the MITC elements stabilize as $N$ is increased. The convergence curves for the displacement-based elements indicate that their respective smallest nonzero eigenvalues rapidly decrease to zero.

Therefore, we conclude that the MITC elements pass the inf-sup test for the free cylinder problem. The displacement-based elements, of course, fail the test.

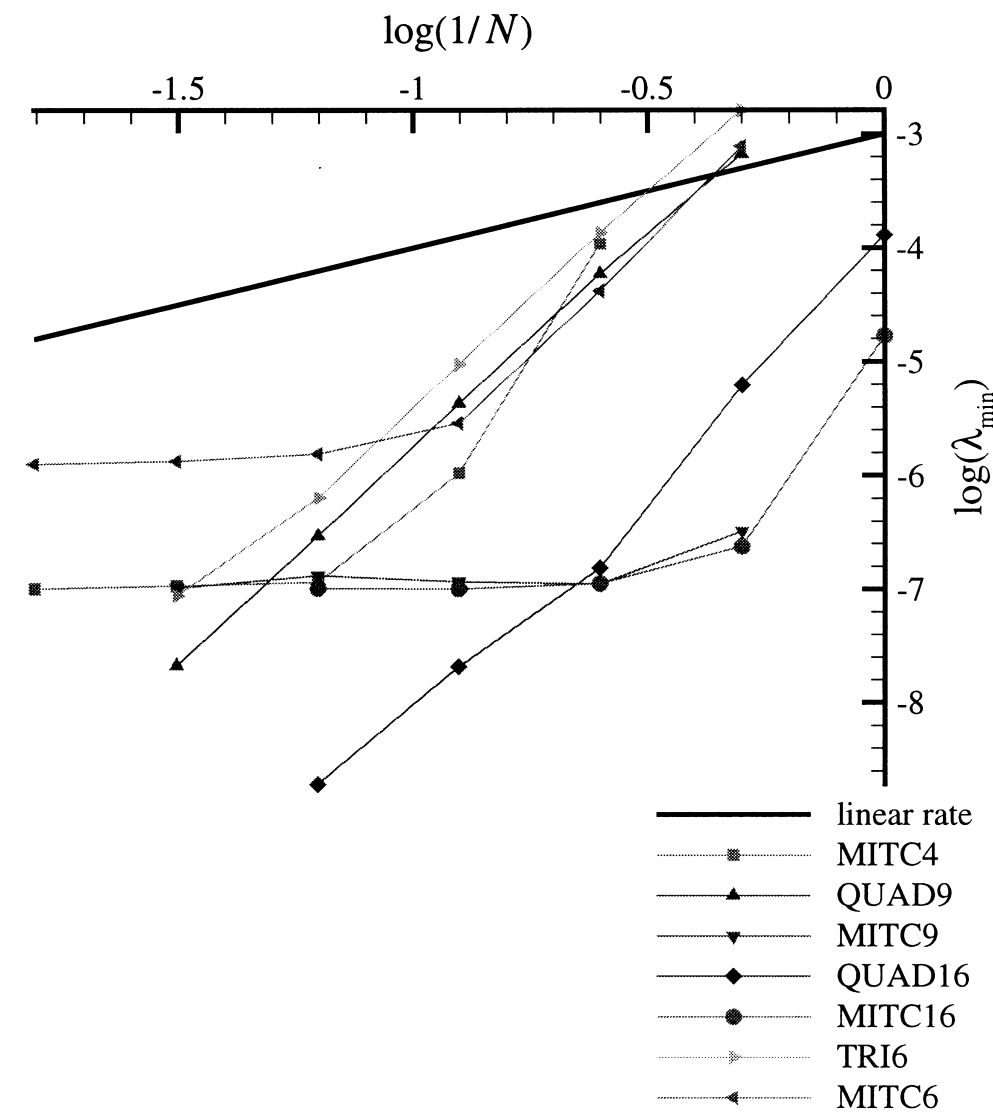

Fig. 4. Inf-sup test. Convergence of the smallest nonzero eigenvalue using uniform meshes of shell elements for the partly clamped hyperbolic paraboloid problem. 


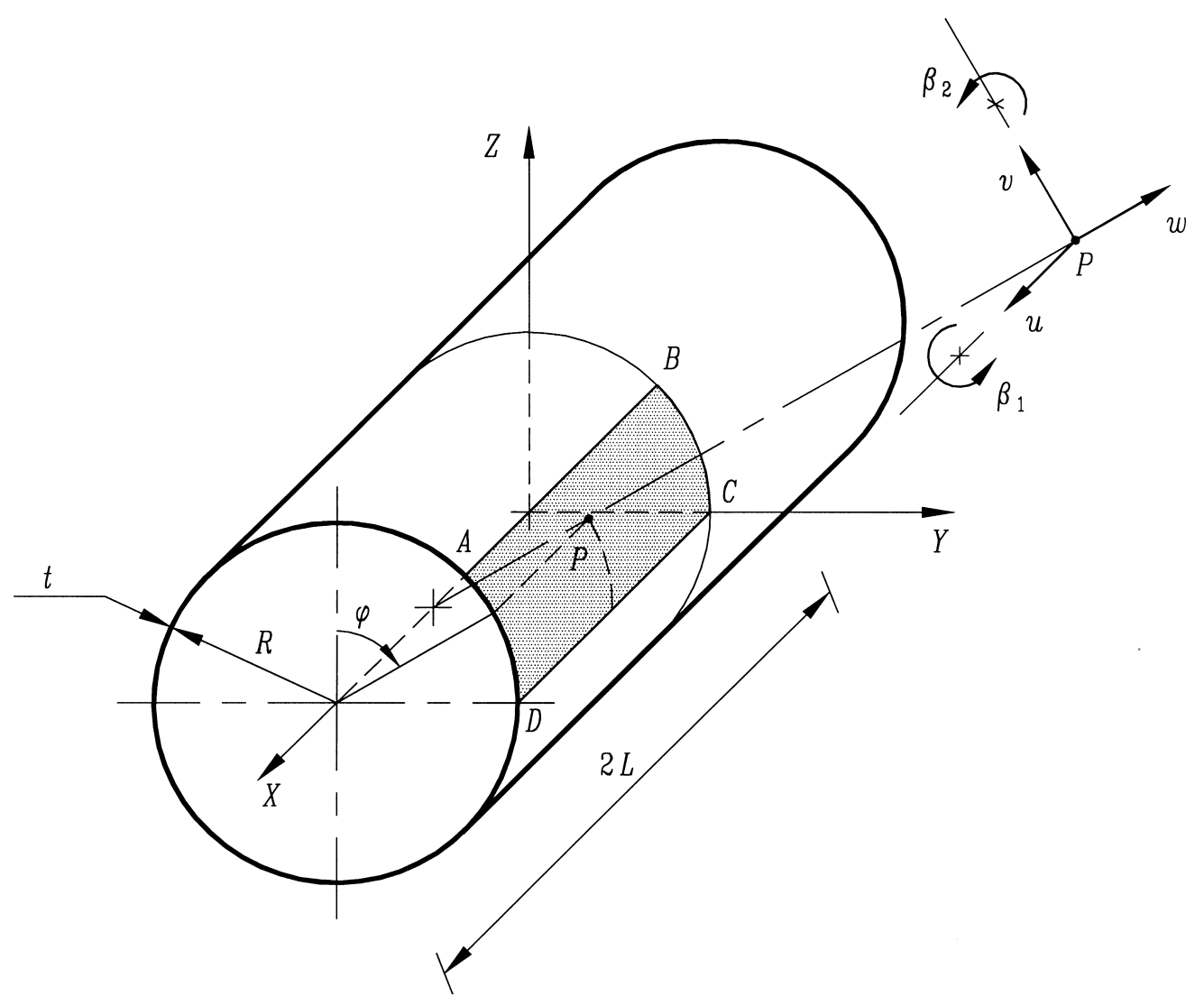

Fig. 5. Cylindrical shell.

\subsection{Plate bending analysis}

Of course, if a general shell element passes the infsup test in plate bending, it is expected to satisfy the plate bending inf-sup condition of Ref. [4]. In this section we address the applicability of our numerical infsup test procedure for the analysis of general shell elements when the plate bending problem is considered.

For our test we consider a fully clamped square plate problem and employ uniform meshes of quadrilateral and triangular elements.

A numerical inf-sup study of displacement-based and mixed-interpolated MITC plate elements has been carried out in [4] and [5]. For comparison with the results presented in those papers, we consider an additional element, the four-node displacement-based general shell element QUAD4.

Fig. 7 displays the convergence of the smallest nonzero eigenvalue.

The $\lambda_{\min }$ convergence curves of the higher-order quadrilateral displacement-based elements, QUAD9 and QUAD16, stabilize in the neighborhood of $10^{-2}$ and hence the elements pass the inf-sup test. The QUAD4 element, however, fails the test as its smallest eigenvalue rapidly converges to zero when the mesh is refined. All the MITC elements pass the test.

Next we consider a sequence of distorted meshes as shown in Fig. 8. Fig. 9 summarizes the results of the inf-sup test. The only drastic change in the elements' behavior compared with the uniform meshes is that the nine-node displacement-based element QUAD9 fails the test. This evidence is consistent with the results of the inf-sup test for plate bending reported in [4] and [5], which show that the nine-node displacement-based quadrilateral element passes the inf-sup test for plate bending when uniform meshes with nondistorted elements are considered and fails when mesh distortions are introduced.

\section{Concluding remarks}

We have developed a simple and effective numerical inf-sup test methodology to identify if a particular 


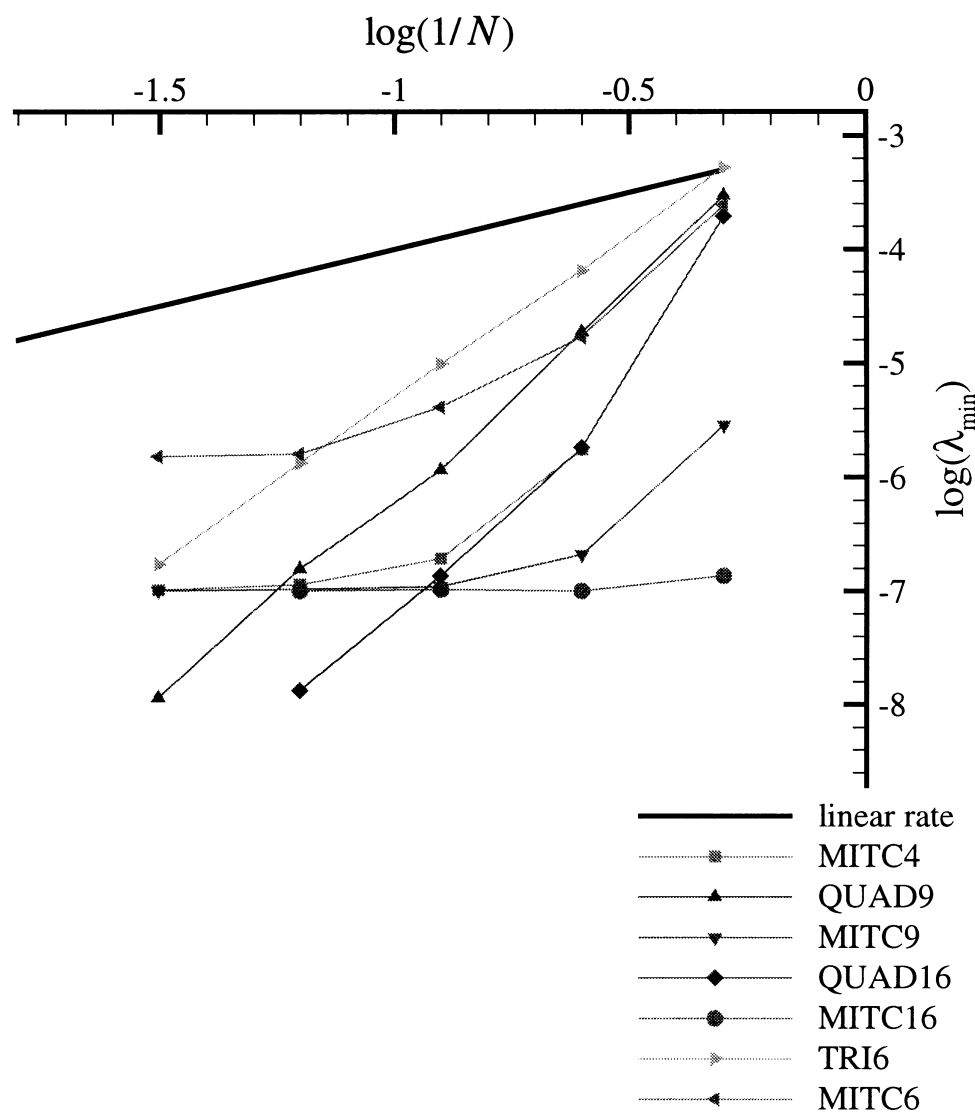

Fig. 6. Inf-sup test. Convergence of the smallest nonzero eigenvalue using uniform meshes of shell elements for the free cylindrical shell problem.

finite element discretization satisfies the inf-sup condition in the analysis of bending-dominated shells.

We have specified details of computer implementation of the proposed procedure and applied it to displacement-based and MITC shell elements.

Based on the study, we can conclude:

- The MITC elements (including the four-node element) have passed all the tests and hence we can expect that they satisfy the inf-sup condition in the analysis of bending-dominated shells.

- The hyperbolic paraboloid shell is a "tough" test problem for the detection of the elements' locking behavior and is most suitable as the benchmark problem in the numerical inf-sup test, see also Ref. [11].

- The proposed numerical inf-sup test procedure proved effective in identifying whether or not general shell elements satisfy the inf-sup condition when used in plate bending analysis.

However, limitations of the proposed test procedure should be kept in mind:
- An element which satisfies the inf-sup condition is not guaranteed to perform well in membrane-dominated problems. Separate convergence studies have to be carried out to establish whether or not the element is optimal in membrane-dominated situations. For example, the mixed-stabilized elements proposed by Arnold and Brezzi [13] were analytically (under simplifying assumptions) shown to satisfy the infsup condition, and yet are known to suffer from poor convergence in membrane-dominated cases [14].

- The evaluation of the discrete inf-sup condition is geometry- and mesh-dependent, with the most important implication being that an element shown to pass the inf-sup test for one geometry and sequence of meshes is not guaranteed to pass for a different geometry and/or different sequence of meshes. The sensitivity of numerical results to the problem geometry is, in general, unavoidable, but has been only a side issue in analyses of incompressible media and plate bending $[1,4]$. In the design of shells, however, 


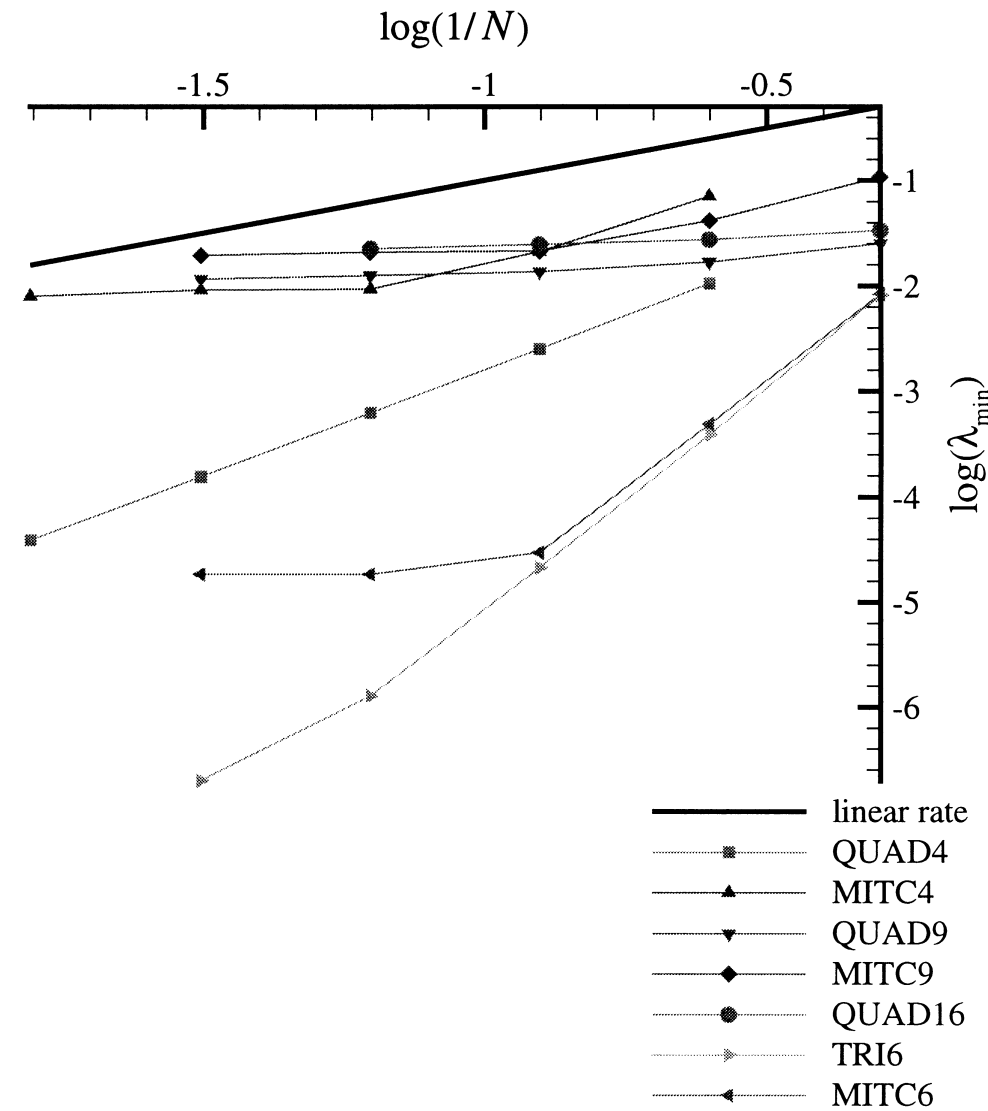

Fig. 7. Inf-sup test. Convergence of the smallest nonzero eigenvalue using uniform meshes of shell elements for the plate bending problem.

the choice of geometry and boundary conditions is the key factor which solely determines the behavior of the shell [10]. Additional sensitivity of the finite element solution to the mesh orientation further limits the generality of numerical studies.

While these limitations are profound, the test proposed in this paper is still very valuable. If this test is passed (on the problems considered in this paper), it is likely that the inf-sup condition is satisfied.

We suggest that current and newly proposed shell and plate finite elements be evaluated using this test.

\section{Appendix A. Summary of MITC shell element interpolations}

Let $\hat{K}$ denote the area of a reference element. Using the notation already defined, the spaces used for the elements are then as follows:

\section{A.1. The MITC4 element}

The following functional spaces are used for the four-node element:

$$
\begin{aligned}
& \mathscr{V}^{h}=\left\{\mathbf{v} \in\left[H^{1}(\Omega)\right]^{3},\left.\mathbf{v}\right|_{\hat{K}} \in\left[Q_{1}(\hat{K})\right]^{3}\right\}, \\
& \mathscr{B}^{h}=\left\{\boldsymbol{\eta} \in\left[H^{1}(\Omega)\right]^{2},\left.\boldsymbol{\eta}\right|_{\hat{K}} \in\left[Q_{1}(\hat{K})\right]^{2}\right\},
\end{aligned}
$$

$\mathscr{E}_{13}^{h}=\left\{\psi \in L^{2}(\Omega),\left.\psi\right|_{\hat{K}} \in \operatorname{span}\left\{1, \hat{\xi}_{2}\right\}\right\}$,

$\mathscr{E}_{23}^{-h}=\left\{\psi \in L^{2}(\Omega),\left.\psi\right|_{\hat{K}} \in \operatorname{span}\left\{1, \hat{\xi}_{1}\right\}\right\}$.

The tying procedure for the element is shown in Fig. A1(A). 


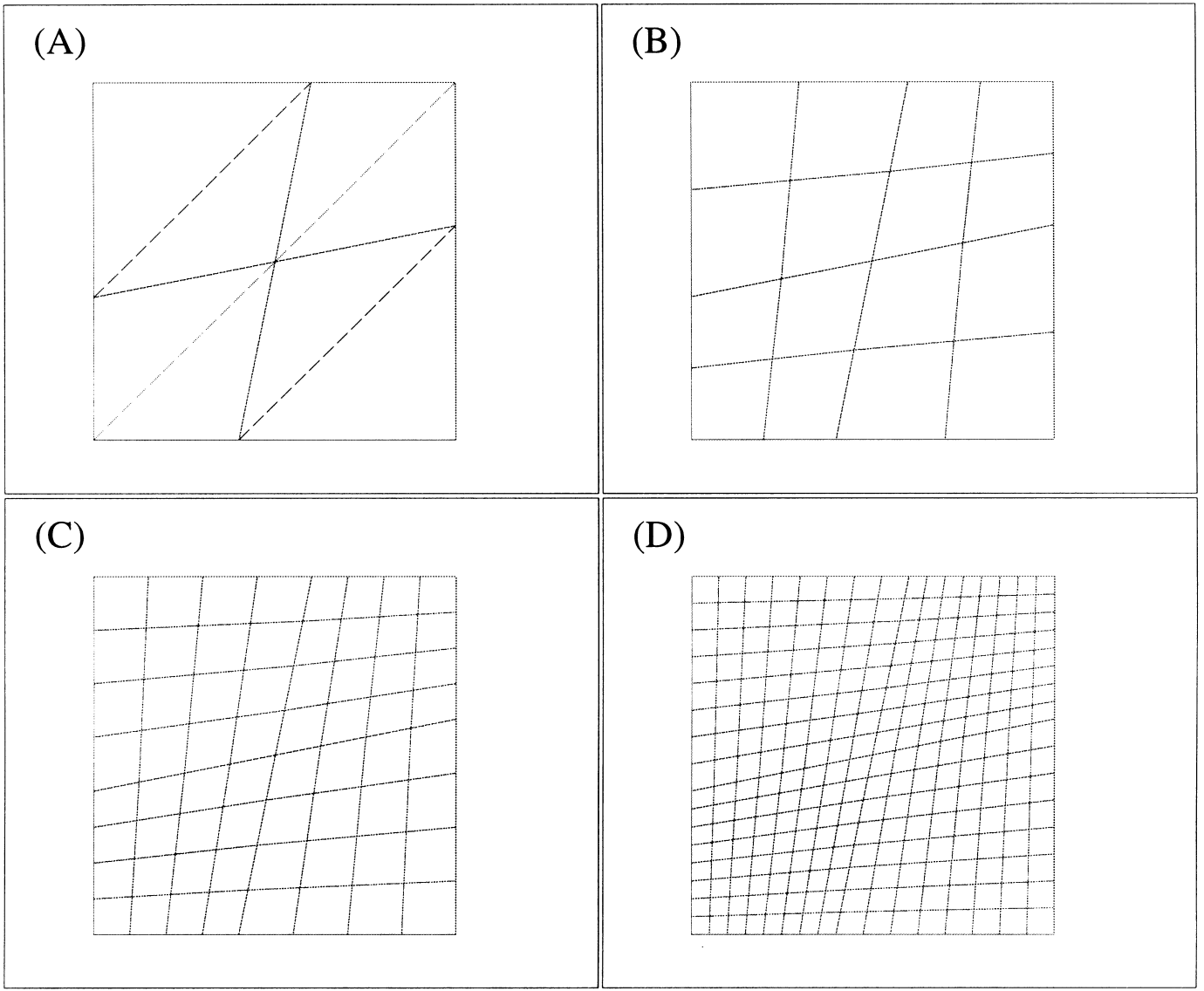

Fig. 8. A sequence of distorted meshes for the plate bending problem. Triangular elements are obtained by subdividing each quadrilateral element as shown in Panel (A).

\section{A.2. The MITC9 element}

The following functional spaces are used to construct the nine-node element:

$\mathscr{V}^{h}=\left\{\mathbf{v} \in\left[H^{1}(\Omega)\right]^{3},\left.\mathbf{v}\right|_{\hat{K}} \in\left[Q_{2}(\hat{K})\right]^{3}\right\}$,

$\mathscr{B}^{h}=\left\{\boldsymbol{\eta} \in\left[H^{1}(\Omega)\right]^{2},\left.\boldsymbol{\eta}\right|_{\hat{K}} \in\left[Q_{2}(\hat{K})\right]^{2}\right\}$,

$\mathscr{E}_{11}^{\mathscr{E}^{h}}=\mathscr{E}_{13}^{-h}=\left\{\psi \in L^{2}(\Omega)\right.$,

$\left.\left.\psi\right|_{\hat{K}} \in \operatorname{span}\left\{1, \hat{\xi}_{1}, \hat{\xi}_{2}, \hat{\xi}_{1} \hat{\xi}_{2}, \hat{\xi}_{2}^{2}, \hat{\xi}_{1} \hat{\xi}_{2}^{2}\right\}\right\}$,
$\mathscr{E}_{22}^{\not h}=\mathscr{E}_{23}^{\text {hh }}=\left\{\psi \in L^{2}(\Omega)\right.$,

$\left.\left.\psi\right|_{\hat{K}} \in \operatorname{span}\left\{1, \hat{\xi}_{1}, \hat{\xi}_{2}, \hat{\xi}_{1} \hat{\xi}_{2}, \hat{\xi}_{1}^{2}, \hat{\xi}_{1}^{2} \hat{\xi}_{2}\right\}\right\}$.

$\mathscr{E}_{12}^{h_{h}}=\left\{\psi \in L^{2}(\Omega),\left.\psi\right|_{\hat{K}} \in Q_{1}(\hat{K})\right\}$.

The tying procedure for the element is given in Fig. A1(B).

\section{A.3. The MITC16 element}

The 16-node cubic element is constructed using

$\mathscr{V}^{h}=\left\{\mathbf{v} \in\left[H^{1}(\Omega)\right]^{3},\left.\mathbf{v}\right|_{\hat{K}} \in\left[Q_{3}(\hat{K})\right]^{3}\right\}$,

$\mathscr{B}^{h}=\left\{\boldsymbol{\eta} \in\left[H^{1}(\Omega)\right]^{2},\left.\boldsymbol{\eta}\right|_{\hat{K}} \in\left[Q_{3}(\hat{K})\right]^{2}\right\}$, 


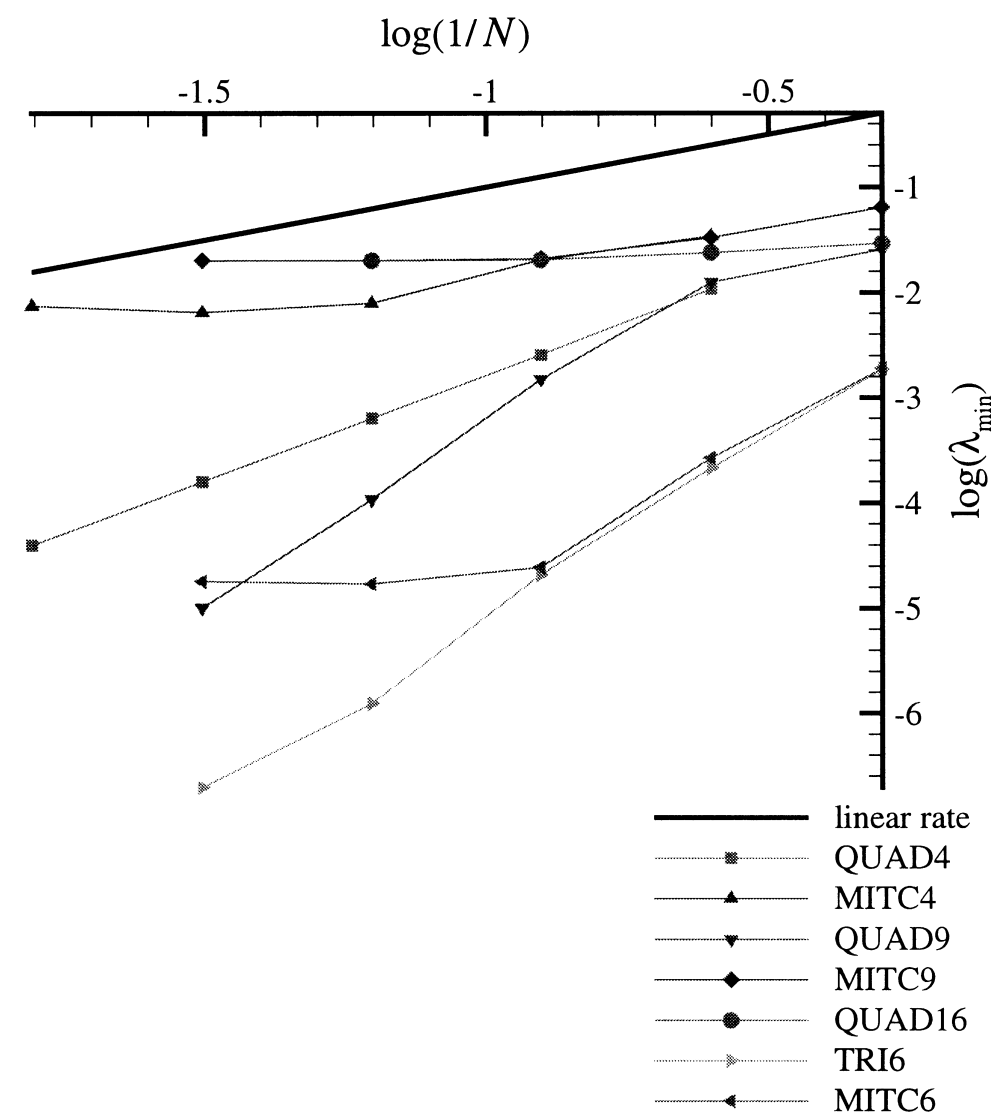

Fig. 9. Inf-sup test. Convergence of the smallest nonzero eigenvalue using distorted meshes of shell elements for the plate bending problem.

$$
\begin{aligned}
& \mathscr{E}_{11}^{h_{1}^{h}}=\mathscr{E}_{13}^{h^{h}}=\left\{\psi \in L^{2}(\Omega),\right. \\
& \left.\psi\right|_{\hat{K}} \in \operatorname{span}\left\{1, \hat{\xi}_{1}, \hat{\xi}_{2}, \hat{\xi}_{1}^{2}, \hat{\xi}_{1} \hat{\xi}_{2}, \hat{\xi}_{2}^{2}, \hat{\xi}_{1}^{2} \hat{\xi}_{2},\right. \\
& \left.\left.\hat{\xi}_{1} \hat{\xi}_{2}^{2}, \hat{\xi}_{2}^{3}, \hat{\xi}_{1}^{2} \hat{\xi}_{2}^{2}, \hat{\xi}_{1} \hat{\xi}_{2}^{3}, \hat{\xi}_{1}^{2} \hat{\xi}_{2}^{3}\right\}\right\},
\end{aligned}
$$$$
\mathscr{E}_{22}^{h h}=\mathscr{E}_{23}^{\mathscr{C}^{h}}=\left\{\psi \in L^{2}(\Omega),\right.
$$$$
\left.\psi\right|_{\hat{K}} \in \operatorname{span}\left\{1, \hat{\xi}_{1}, \hat{\xi}_{2}, \hat{\xi}_{1}^{2}, \hat{\xi}_{1} \hat{\xi}_{2}, \hat{\xi}_{2}^{2}, \hat{\xi}_{1}^{3}, \hat{\xi}_{1}^{2} \hat{\xi}_{2},\right.
$$$$
\left.\left.\hat{\xi}_{1} \hat{\xi}_{2}^{2}, \hat{\xi}_{1}^{3} \hat{\xi}_{2}, \hat{\xi}_{1}^{2} \hat{\xi}_{2}^{2}, \hat{\xi}_{1}^{3} \hat{\xi}_{2}^{2}\right\}\right\} \text {. }
$$$$
\mathscr{E}_{12}^{\mathscr{h}_{h}}=\left\{\psi \in L^{2}(\Omega),\left.\psi\right|_{\hat{K}} \in Q_{2}(\hat{K})\right\}
$$

The tying procedure for the strain tensor components is presented in Fig. A1(C).

\section{A.4. The MITC6 element}

The six-node triangular element is constructed using $\mathscr{V}^{h}=\left\{\mathbf{v} \in\left[H^{1}(\Omega)\right]^{3},\left.\mathbf{v}\right|_{\hat{K}} \in\left[P_{2}(\hat{K})\right]^{3}\right\}$,

$$
\mathscr{B}^{h}=\left\{\boldsymbol{\eta} \in\left[H^{1}(\Omega)\right]^{2},\left.\boldsymbol{\eta}\right|_{\hat{K}} \in\left[P_{2}(\hat{K})\right]^{2}\right\},
$$

$\mathscr{E}_{11}^{h_{h}}=\mathscr{E}_{12}^{h_{h}}=\mathscr{E}_{22}^{h_{h}}=\left\{\psi \in L^{2}(\Omega),\left.\psi\right|_{\hat{K}} \in P_{1}(\Omega)\right\}$,

$\mathscr{E}_{13}^{\mathscr{C}^{h}}=\left\{\psi \in L^{2}(\Omega),\left.\psi\right|_{\hat{K}} \in \operatorname{span}\left\{1, \hat{\xi}_{1}, \hat{\xi}_{1} \hat{\xi}_{2}, \hat{\xi}_{2}, \hat{\xi}_{2}^{2}\right\}\right\}$,

$\mathscr{E}_{23}^{\mathscr{C}^{h}}=\left\{\psi \in L^{2}(\Omega),\left.\psi\right|_{\hat{K}} \in \operatorname{span}\left\{1, \hat{\xi}_{1}, \hat{\xi}_{1} \hat{\xi}_{2}, \hat{\xi}_{2}, \hat{\xi}_{1}^{2}\right\}\right\}$.

This choice of interpolation spaces for the transverse shear components proved to be optimal for the mixed- 
(A)

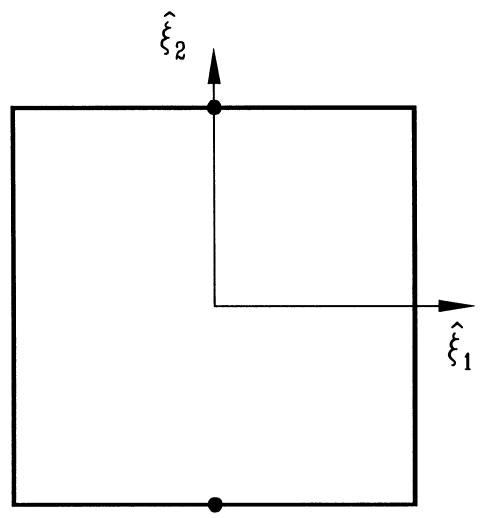

(C)

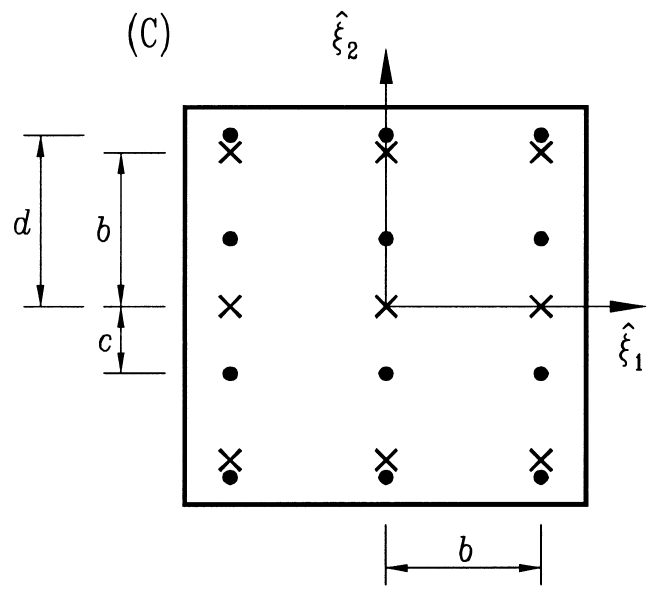

(B)

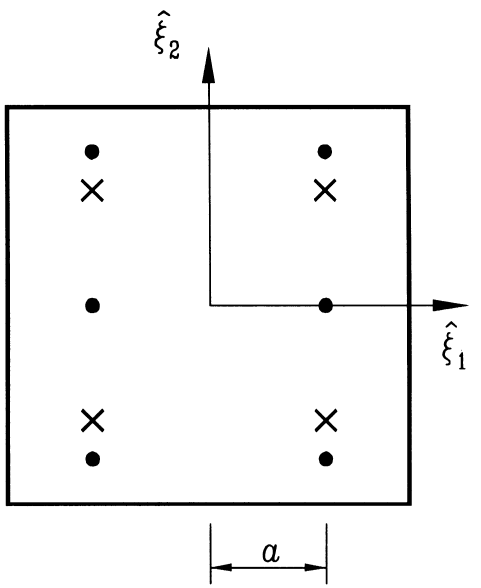

- component $\varepsilon_{13}(\mathrm{~A})$;

components $\varepsilon_{11}, \varepsilon_{13}$ (B and $\mathrm{C}$ )

$\times$ component $\varepsilon_{12}$

$a=0.577 \ldots$

$b=0.775 \ldots$

$c=0.339 \ldots$

$d=0.861 \ldots$

Fig. A1. Tying points for the MITC shell elements. (A) MITC4 element; (B) MITC9 element; (C) MITC16 element.

interpolated seven-node plate bending element MITC7 [1]. The tying procedure for the strain tensor components is depicted in Fig. A2.

\section{Appendix B. Proof of Proposition 2}

1. Assume that Eq. (41) holds.

Then, of course, Eq. (49) holds too. Next, to prove $(\mathscr{A})$ take $\boldsymbol{\Psi} \in \mathscr{E}^{h}$ such that

$B(\boldsymbol{\Psi}, \mathbf{V})=0, \quad \forall \mathbf{V} \in \mathscr{U}^{h}$

From Eq. (41), we get

$$
\sup _{\mathbf{V} \in \mathscr{U}} \frac{B(\boldsymbol{\Psi}, \mathbf{V})}{\|\mathbf{V}\|_{\mathscr{U}}}=0
$$

hence

$$
B(\boldsymbol{\Psi}, \mathbf{V})=0, \quad \forall \mathbf{V} \in \mathscr{U},
$$

so that $(\mathscr{A})$ holds too.

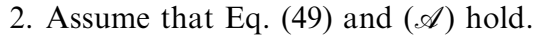

To prove that Eq. (41) holds, consider any $\boldsymbol{\Psi} \in$ $\mathscr{E}^{h}$. It can be uniquely decomposed into

$\boldsymbol{\Psi}=\boldsymbol{\Psi}_{0}+\boldsymbol{\Psi}_{1}$,

where $\boldsymbol{\Psi}_{0} \in \operatorname{Im} B^{h}$ and $\boldsymbol{\Psi}_{1}$ is in the subspace of $\mathscr{E}^{h}$ $L^{2}$-orthogonal to $\operatorname{Im} B^{h}$, i.e.

$C\left(\boldsymbol{\Psi}_{1}, \boldsymbol{\Theta}\right)=0, \quad \forall \boldsymbol{\Theta} \in \operatorname{Im} B^{h}$.

According to the definition of $\operatorname{Im} B^{h}$ given in Eq. (47), this is equivalent to

$B\left(\boldsymbol{\Psi}_{1}, \mathbf{W}\right)=0, \quad \forall \mathbf{W} \in \mathscr{U}^{h}$,

hence, from $(\mathscr{A})$, also equivalent to

$B\left(\boldsymbol{\Psi}_{1}, \mathbf{W}\right)=0, \quad \forall \mathbf{W} \in \mathscr{U}$. 

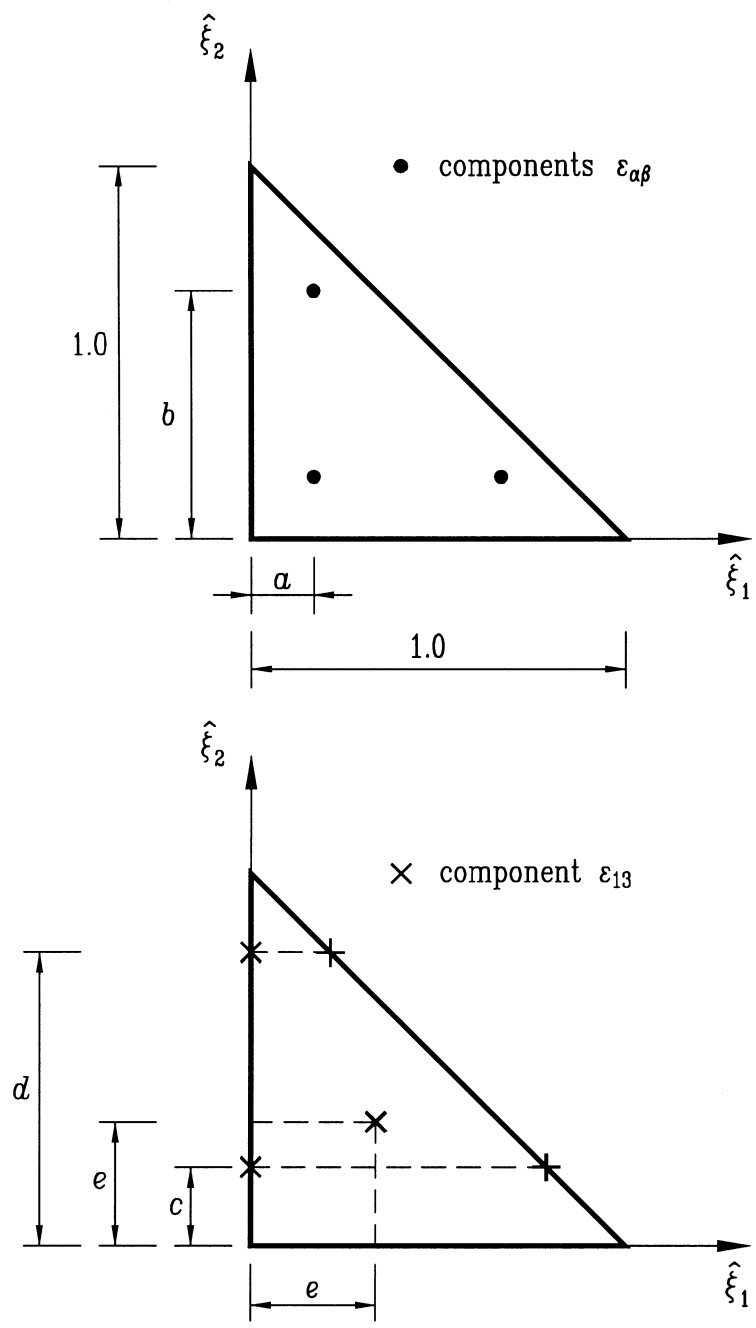

$$
\begin{aligned}
& a=0.166 \ldots \\
& b=0.666 \ldots \\
& c=0.211 \ldots \\
& d=0.788 \ldots \\
& e=0.333 \ldots
\end{aligned}
$$

Fig. A2. Tying points for the six-node mixed-interpolated shell element MITC6.

Using this and Eq. (49), we get

$$
\begin{aligned}
& \sup _{\mathbf{V} \in \mathscr{U}^{h}} \frac{B(\boldsymbol{\Psi}, \mathbf{V})}{\|\mathbf{V}\|_{\mathscr{U}}}=\sup _{\mathbf{V} \in \mathscr{U}^{h}} \frac{B\left(\boldsymbol{\Psi}_{0}, \mathbf{V}\right)}{\|\mathbf{V}\|_{\mathscr{U}}} \\
& \geq c \sup _{\mathbf{V} \in \mathscr{U}} \frac{B\left(\boldsymbol{\Psi}_{0}, \mathbf{V}\right)}{\|\mathbf{V}\|_{\mathscr{U}}} \\
& =c \sup _{\mathbf{V} \in \mathscr{U}} \frac{B(\boldsymbol{\Psi}, \mathbf{V})}{\|\mathbf{V}\|_{\mathscr{U}}} .
\end{aligned}
$$

Therefore, Eq. (41) holds.

This completes the proof of Proposition 2.

\section{References}

[1] Bathe KJ. Finite element procedures. Englewood Cliffs, NJ: Prentice-Hall, 1996

[2] Brezzi F, Fortin M. Mixed and hybrid finite element methods. Berlin: Springer-Verlag, 1991.

[3] Chapelle D, Bathe KJ. The inf-sup test. Computers and Structures 1993;47:537-45.

[4] Iosilevich A, Bathe KJ, Brezzi F. On evaluating the infsup condition for plate bending elements. Int $\mathbf{J}$ Num Meth Eng 1997;40:3639-63.

[5] Iosilevich A, Bathe KJ, Brezzi F. Numerical inf-sup analysis of MITC plate bending elements. In: Proc. of the American Math. Society, Symposium on Plates and Shells. Université Laval. 1996. 
[6] Dvorkin EN, Bathe KJ. A continuum mechanics based four-node shell element for general nonlinear analysis. Eng Comput 1984;1:77-88.

[7] Bucalem ML, Bathe KJ. Higher-order MITC general shell elements. Int J Num Meth Eng 1993;36: 3729-54.

[8] Chapelle D, Bathe KJ. On general shell finite elements and mathematical shell models. In: Topping BHV, editor. Advances in finite element procedures and techniques. Edinburgh, Scotland: Civil-Comp Press, 1998. p. 25-30.

[9] Naghdi PM. Foundations of elastic shell theory. In: Progress in solid mechanics, vol. 4. Amsterdam: NorthHolland, 1963. p. 1-90.

[10] Chapelle D, Bathe KJ. Fundamental considerations for the finite element analysis of shell structures. Computers and Structures 1998;66:19-36.
[11] Bathe KJ, Iosilevich A, Chapelle D. An evaluation of the MITC shell elements. Computers and Structures, in press.

[12] Brezzi F, Bathe KJ. A discourse on the stability conditions for mixed finite element formulations. Comp Meth Appl Mech Eng 1990;82:27-57.

[13] Arnold DN, Brezzi F. Locking-free finite element methods for shells. Math Comp 1997;66:1-14.

[14] Bucalem ML, da Nobrega SHS. A mixed formulation for general triangular isoparametric shell elements based on the degenerated solid approach. In: Topping BHV, editor. Advances in finite element procedures and techniques. Edinburgh, Scotland: Civil-Comp Press, 1998. p. 53-62.

[15] Chapelle D, Stenberg R. Stabilized finite element formulations for shells in a bending dominated state. SIAM J Num Anal 1998;36(1):32-73. 